\title{
Ebselen reduces cigarette smoke-induced vascular endothelial dysfunction in mice
}

\author{
Kurt Brassington ${ }^{1}$, Stanley Chan ${ }^{1}$, Huei Seow ${ }^{1}$, Aleksandar Dobric ${ }^{1}$, Steven Bozinovski ${ }^{1}$, \\ Stavros Selemidis ${ }^{1}$, and Ross Vlahos ${ }^{1}$ \\ ${ }^{1}$ RMIT University
}

September 11, 2020

\begin{abstract}
Background and Purpose: It is well established that both smokers and patients with COPD are at a significantly heightened risk of cardiovascular disease (CVD), although the mechanisms underpinning the onset and progression of comorbid CVD are largely unknown. Here, we explored whether cigarette smoke (CS) exposure impairs vascular function in mice and given the well-known pathological role for oxidative stress in COPD, whether the antioxidant compound ebselen prevents CS-induced vascular dysfunction in mice. Experimental Approach: Male BALB/c mice were exposed to either room air (sham) or CS generated from 9 cigarettes per day, 5 days a week for 8 weeks. Mice were treated with ebselen $(10 \mathrm{mg} / \mathrm{kg}$, oral gavage once daily) or vehicle $(5 \% \mathrm{w} / \mathrm{v}$ CM cellulose in water) $1 \mathrm{~h}$ prior to the first CS exposure of the day. Upon sacrifice, bronchoalveolar lavage fluid (BALF) was collected to assess pulmonary inflammation and the thoracic aorta was excised to investigate vascular endothelial and smooth muscle dilator responses ex-vivo. Key Results: CS exposure caused a significant increase in lung inflammation which was reduced by ebselen. CS also caused significant endothelial dysfunction in the thoracic aorta which was attributed to a downregulation of eNOS expression and increased vascular oxidative stress. Ebselen abolished the aortic endothelial dysfunction seen in CS-exposed mice by reducing the oxidative burden and preserving eNOS expression. Conclusion and Implications: Targeting CS-induced oxidative stress with ebselen may provide a novel means for treating the life-threatening pulmonary and cardiovascular manifestations associated with cigarette smoking and COPD.
\end{abstract}

\section{Introduction}

Chronic obstructive pulmonary disease (COPD) is a major incurable global health burden and is currently the $4^{\text {th }}$ largest cause of death in the world (WHO, 2018). Approximately $50 \%$ of COPD patients will die from a cardiovascular event (Sin \& Man, 2005; TLF, 2017) and consequently the pathobiological mechanisms linking COPD to cardiovascular disease are now an area of intensive research. Each puff of cigarette smoke contains $>10^{16}$ free radicals per puff, driving oxidative stress and tissue damage (Bartalis, Chan \& Wooten, 2007) This oxidative stress and inflammation, have been shown to alter pulmonary blood vessel structure, through driving vascular remodelling, and promoting arterial stiffness and atherosclerosis (Sin, Anthonisen, Soriano \& Agusti, 2006). Vascular tone is controlled by vasoactive substances such as NO and prostaglandins, their secretion which can be maintained by circulating oxygen levels (Chan \& Vanhoutte, 2013). Under hypoxic conditions like those seen in COPD, dysregulation of this vascular homeostatic balance occurs, due to oxidative damage to the vascular endothelial cells (VECs) leading to impaired NO production, thereby promoting endothelial dysfunction (Chan \& Vanhoutte, 2013). NO is a key vasodilator produced by endothelial nitric oxide synthase (eNOS). Under normal physiological conditions, increased sheer stress on the vascular endothelium stimulates mechanosensitive ion channels, triggering a rapid influx of $\mathrm{Ca}^{2+}$ into the cytoplasm of the VECs. This increases eNOS activity via myoendothelial gap junctions, that transmit vasodilatory NO signals to the underlying smooth muscle cells. VECs appear to be sensitive to oxidative 
damage, which may be the result of the conversion of $\mathrm{NO}$ to peroxynitrite $\left(\mathrm{ONOO}^{-}\right)$in the presence of the harmful ROS; superoxide $\left(\mathrm{O}_{2}^{-}\right)$that ultimately reduces vascular NO bioavailability (Endemann \& Schiffrin, 2004; Kolluru, Bir \& Kevil, 2012; Tabit, Chung, Hamburg \& Vita, 2010). An altered oxidative balance in VECs has been demonstrated to promote cardiovascular manifestations such as atherosclerosis, myocardial infarction (MI) and stroke (Brassington, Selemidis, Bozinovski \& Vlahos, 2019; Endemann \& Schiffrin, 2004; Kolluru, Bir \& Kevil, 2012; Tabit, Chung, Hamburg \& Vita, 2010).

Although cardiovascular comorbidities are the largest cause of mortality in COPD, the detrimental effects of CS and its associated oxidative stress on the systemic vasculature remain largely unknown. Given the deleterious role of oxidative stress in COPD, antioxidant treatment may be a viable therapeutic approach to treat the cardiovascular manifestations associated with this disease. We have previously shown that the antioxidant ebselen (2-phenyl-1,2-benzisoselenazol-3(2H)one), an organoselenium glutathione peroxidase (Gpx) mimetic, inhibits CS-induced lung inflammation in mice (Duong, Seow, Bozinovski, Crack, Anderson \& Vlahos, 2010). Ebselen treatment may also be an effective therapeutic in chronic diseases such as atherosclerosis, thrombosis, and stroke where oxidative stress and inflammation play a crucial role (Azad \& Tomar, 2014; Sarker et al., 2003; Sarma \& Mugesh, 2008; Takasago, Peters, Graham, Masayasu \& Macrae, 1997). Moreover, studies have shown that Gpx-1 deficient mice have enhanced pulmonary inflammation (Duong, Seow, Bozinovski, Crack, Anderson \& Vlahos, 2010), as well as worsened cardiovascular outcomes including a larger infarct volume following ischemic stroke (Crack et al., 2001; Duong, Seow, Bozinovski, Crack, Anderson \& Vlahos, 2010), suggesting that Gpx-1 (or compounds which mimic its actions, like ebselen) may exhibit protective effects.

Of interest, Gpx-1 activity has been shown to be elevated in smokers as a potential mechanism to counteract the harmful oxidative stress. However, Gpx-1 has been reported to be severely depleted in the lungs of COPD patients, resulting in an overstated inflammatory response and oxidative burden (Kluchova, Petrasova, Joppa, Dorkova \& Tkacova, 2007; Santos et al., 2004; Tkacova, Kluchova, Joppa, Petrasova \& Molcanyiova, 2007; Vlahos et al., 2010). A study by Chew et.al has showcased the therapeutic effect of ebselen in Gpx-1 knockout mice, with the study finding that synthetic repletion of Gpx activity in these diabetic mice produced atheroprotective effects in vivo (Chew et al., 2010). Exogenous repletion of Gpx-1 with compounds like ebselen may have therapeutic potential in not only treating the pulmonary manifestations of COPD but perhaps its cardiovascular comorbidities.

In the present study we investigated whether chronic CS exposure in a preclinical mouse model of COPD impairs vascular function and whether ebselen can prevent CS-induced vascular dysfunction in mice.

\section{Methods}

\section{Animals}

All experiments were conducted in accordance with the Australian Code of Practice for the Care of Experimental Animals, the ARRIVE Guidelines and with RMIT University Animal Ethics Committee approval (Animal Ethics Application Number 1521). 7-week-old male Balb/c mice ( $20 \mathrm{~g}$ body weight) were obtained from the Animal Resource Centre Pty. Ltd (Perth, Australia). Mice were housed in micro-isolator cages (Able Scientific, Australia) at $21^{\circ} \mathrm{C}$ with ad libitum access to water and standard mouse chow (Glen Forest Speciality Foods, Australia).

\section{Cigarette smoke exposure \& ebselen treatment}

Mice were placed into an 18-litre Perspex chamber (The Plastic Man, Huntingdale, Victoria, Australia) in a standard fume cabinet (Aircare Extraction Systems LTD, Clayton, Victoria, Australia) and exposed to cigarette smoke generated from 9 Winfield Red Cigarettes (total particulate matter of $419 \mathrm{mg} \mathrm{m}^{-3}, 16 \mathrm{mg}$ or less of tar, $1.2 \mathrm{mg}$ or less of nicotine and $15 \mathrm{mg}$ or less of CO, Philip Morris, Moorabbin, Australia) for 5 days a week for 8 weeks. Mice were exposed to CS delivered 3 times per day with 3 cigarettes delivered at $9 \mathrm{AM}, 12$ noon and $3 \mathrm{PM}$, over a $1 \mathrm{~h}$ time-period. CS was generated in $60 \mathrm{~mL}$ tidal volumes over $10 \mathrm{~s}$, via a timed draw-back to mimic normal smoking inhalation and burn rates. We have previously shown that this 
CS exposure protocol in Balb/C mice replicates key clinical traits of early stage COPD in humans, including lung inflammation and pathology (mucous hypersecretion and impaired lung function), increased lung and systemic oxidative stress and comorbidities including skeletal muscle wasting (Austin, Crack, Bozinovski, Miller \& Vlahos, 2016; Chan et al., 2019; Vlahos \& Bozinovski, 2014). Thus, this model provides a robust clinically relevant platform to test therapies for COPD and its comorbidities. Sham-exposed mice are placed into an identical 18-liter Perspex chamber, but do not receive cigarette smoke. Mice were weighed every second day (prior to initial CS exposure) up to and including the day of cull. For the ebselen treatment studies, mice were administered $10 \mathrm{mg} \mathrm{kg}^{-1}$ of ebselen (Sapphire Bioscience, Australia) prepared in 5\% w/v CM-cellulose in water (Sigma-Aldrich, USA) or vehicle treated with 5\% CM-cellulose in water alone. Treatments were administered via oral gavage once daily, $1 \mathrm{~h}$ prior to the initial CS exposure.

\section{Bronchoalveolar lavage and lung collection}

Animals were euthanised at the end of the experimental protocol via intraperitoneal injection of sodium pentobarbitone (240 $\mathrm{mg} \mathrm{kg}^{-1}$; Virbac, NSW, Australia). Lungs were then lavagedin situ via a surgical tracheotomy with $0.4 \mathrm{~mL}$ of chilled PBS initially followed by $0.3 \mathrm{~mL}$ PBS thrice, with $\sim 1 \mathrm{~mL}$ of BAL fluid (BALF) retrieved per mouse as previously described (Vlahos et al., 2006) . Total viable cell numbers in the BALF were determined using 50 $\mu \mathrm{L}$ of BALF diluted with $50 \mu \mathrm{l}$ of acridine orange/ethidium bromide $(\mathrm{AO} / \mathrm{EB})$ (Invitrogen, USA). Cell counting was carried out using a standard Neubauer haemocytometer, under fluorescent light on an Olympus BX53 microscope (Olympus, Japan). Right ventricular perfusion with 6-7 $\mathrm{mL}$ of PBS was then performed to clear whole lungs from blood, and the lungs then excised, rinsed in PBS, snap-frozen in liquid nitrogen and stored at $-80^{\circ} \mathrm{C}$ until required.

\section{Differential cell counting}

To differentiate the various cell populations in the BALF, cytocentrifuged cell preparations (Shandon Cytospin 3, $400 \mathrm{RPM}, 10 \mathrm{~min}$ ) were used, with $\sim 5 \times 10^{4}$ cells per slide. Dried cytospots were subjected to a Shandon Kwik-Diff ${ }^{\circledR}$ Fixative (Thermo Fischer Scientific, USA), followed by Hemacolour ${ }^{\circledR}$ eosin and thiazine differential stains (Merck, USA) as outlined in the manufacturer's instructions. Cell types (i.e. macrophages, neutrophils and lymphocytes) were identified according to standard morphological criteria, using the above-mentioned microscope, with at least 500 cells counted per slide.

\section{Quantitative real-time PCR (RT-qPCR)}

Total RNA was extracted from approximately $10 \mathrm{mg}$ of whole lung tissue using a RNeasy ${ }^{\circledR}$ Mini Kit (Qiagen, Germany). Isolated mRNA was then reverse transcribed with a High Capacity RNA-to-cDNA kit (Thermo Fisher Scientific, USA). Real time PCR reactions were performed using Thermo Fisher Scientific pre-developed Taqman assay reagents in triplicate using GAPDH as the internal housekeeping control. Through utilisation of the threshold cycle $\left(\mathrm{C}_{t}\right)$ value which is the PCR cycle number out of 40 at which the fluorescence signal measured exceeds the calculated background threshold, indicative of amplification of the target sequence value, which is proportional to the number of target copies within the sample and converting this result to the threshold cycle time ([?][?]CT). mRNA expression levels can then be quantified and referenced against GAPDH, allowing comparisons between treatment groups to be made.

\section{Vascular reactivity}

To assess the effect of both CS-exposure and ebselen treatment on vessel function, thoracic aorta was excised from the mice and all perivascular fat removed. Vessels were placed into carbogen-bubbled $\left(95 \% \mathrm{O}_{2}, 5 \%\right.$ $\mathrm{CO}_{2}$ ) cold Krebs buffer (composition in mmol/L: $\mathrm{NaCl} 119, \mathrm{KCl} 4.7, \mathrm{MgSO}_{4} 1.17, \mathrm{NaHCO}_{3} 25, \mathrm{KH}_{2} \mathrm{PO}_{4}$ $1.18, \mathrm{CaCl}_{2} 2.5$, glucose 5.5). The aortae were cut into four $2 \mathrm{~mm}$ rings and mounted onto the pins of the myograph system (Danish Myo Technology A/S, Model 610M) with the resting tension increased to $5 \mathrm{mN}$ which was determined to give an effective arterial wall pressure of $\sim 100 \mathrm{mmHg}(13.3 \mathrm{kPa})$ thus mimicking in vivoconditions. Following a 30-minute equilibration, aortic rings were exposed to $0.5 \times 10^{-3} \mathrm{M}$ of the thromboxane A2 agonist U46619 (Cayman Chemical, USA) to induce the maximal vascular contraction, defined as $100 \%$. In each ring, both endothelial integrity and smooth muscle function were assessed using cumulative 
doses of acetylcholine (ACh, $1 \times 10^{-8} \mathrm{M}$ to $1 \times 10^{-5} \mathrm{M}$ ) (Thermo Fisher Scientific, USA) and sodium nitroprusside (SNP, $1 \times 10^{-8} \mathrm{M}$ to $1 \times 10^{-5} \mathrm{M}$ ) (Thermo Fisher Scientific, USA) respectively in sub-maximally contracted aorta (50-60\% of maximal U46619 contraction) with all experiments ran in duplicate and compared to sham or sham + vehicle treated mice.

\section{Immunohistochemical staining for endothelial nitric oxide synthase and oxidative stress}

Vascular expression of endothelial nitric oxide synthase was quantified using eNOS/NOS3 antibody (1:100 dilution, Thermo Fisher Scientific, USA), which is the key nitric oxide generating enzyme within the vascular endothelium. Vascular oxidative stress was measured using 3-Nitrotyrosine (3-NT) (1:100 dilution, Thermo Fisher Scientific, USA) as this is a specific marker for peroxynitrite production, the direct product of the reaction between superoxide to nitric oxide. Thoracic aorta was collected upon cull and fixed in $4 \%$ paraformaldehyde prior to sucrose saturation. The aortae were then paraffin embedded and $4 \mu \mathrm{M}$ sections were cut. Aortic sections were subject to standard deparaffinisation and rehydration, followed by antigen retrieval (in 10mM citric acid, $0.05 \%$ Tween 20, pH 6.0) and blocking (blocking buffer; $10 \%$ horse serum, $10 \%$ FBS, $2 \%$ triton-X, 1xPBS to $50 \mathrm{~mL}$ for $1 \mathrm{hr}$ ). Sections were then incubated overnight with either eNOS of 3-NT primary antibodies at $4 \mathrm{C}$. Excessive primary antibodies were removed by washing, the sections were then incubated at room temperature with the fluorescently labelled secondary antibody, Alexa 488 (1:200 dilution, Thermo Fisher Scientific, USA), then cover slipped using Fluoromount-G ${ }^{\text {TM }}$, with DAPI (Thermo Fisher Scientific, USA) prior to imaging on an Olympus slide scanner VS120-SS (Olympus, Japan). The expression of eNOS and 3-NT was quantified in the endothelial layer of the aortae using Olympus cellSens Dimension $^{\mathrm{TM}}$ desktop software, calculating Object Area Fraction ROI (\%) (version 1:18, Olympus Corporation). All analysis of immunofluorescence was completed in a blinded manner.

\section{Data and Statistical analysis}

All data and statistical analysis comply with the recommendations on experimental design and analysis in pharmacology (Curtis et al, 2018). All data presented as mean \pm standard error of the mean (SEM) unless otherwise stated. Statistical differences between treatments were determined by analysis of variance (ANOVA) followed by Tukey's multiple comparison post-hoc tests where appropriate. One-way ANOVA were used for three of more unmatched groups. Two-way ANOVA were used to analyse data when response was influenced by two categorized factors of interest. For comparisons between two groups of results, regular unpaired t-tests were used to generate the $\mathrm{P}$ value. All statistical analyses were performed using GraphPad Prism $^{\text {TM }}$ for Microsoft Windows@ (Versions 8, Graphpad software ${ }^{\circledR}$, USA) where $p<0.05$ was accepted as significant for all cases.

\section{Materials}

The Suppliers of the following compounds are as follows: Winfield red cigarettes (Phillip Morris, Australia); ebselen (Sapphire Bioscience, Australia); Carboxymethyl Cellulose (Sigma-Aldrich, USA); sodium pentabarbitone (Virbac, Australia); acridine orange/ethidium bromide (Invitrogen, USA); Kwik-Diff ${ }^{\circledR}$ reagent 1, fixative (Thermo Fisher Scientific, USA); Hemacolour ${ }^{\circledR}$ rapid staining of blood smear (eosin solution) (Merck, USA); Hemacolour ${ }^{\circledR}$ rapid staining of blood smear (thiazine solution) (Merck, USA); RNeasy Mini Kit (Qiagen, Germany); High Capacity RNA-to-cDNA kit (Thermo Fisher Scientific, USA); pre-developed TaqMan primers (Thermo Fisher Scientific, USA), CRP ELISA Kit (RayBiotech, USA); U46619 (Cayman Chemical, USA); acetylcholine (Thermo Fisher, USA); sodium nitroprusside (Thermo Fisher, USA); NOS3/eNOS RRID: AB_2533121 (Thermo Fisher Scientific, USA), 3-NT RRID: AB110282 (Abcam, USA); Goat anti-mouse IgG $(\mathrm{H}+\mathrm{L})$ secondary antibody, Alexa Flour Plus 488 RRID: AB_2633275 (Thermo Fisher Scientific, USA); Fluoromount-G ${ }^{\text {TM }}$, with DAPI (Thermo Fisher Scientific, USA) .

\section{Results}

Chronic CS exposure causes endothelial dysfunction in mouse thoracic aorta

The effect of chronic CS exposure on vascular function was examined to establish if the enhanced oxidative stress and inflammation arising from smoking has detrimental effects on blood vessel function in vivo. ACh 
$\left(10^{-8}\right.$ to $\left.10^{-5} \mathrm{M}\right)$ caused an $\sim 90 \%$ maximal relaxation $\left(\mathrm{R}_{\max }\right)$ of $\mathrm{U} 46619$-precontracted thoracic aorta obtained from sham-exposed mice (Figure 1A). However, in thoracic aorta taken from CS-exposed mice, endothelialdependent vasodilatory responses were significantly impaired ( $\sim 40 \% \mathrm{R}_{\max }, p<0.05$ ) demonstrating that chronic CS exposure causes endothelial dysfunction. Sodium nitroprusside $\left(10^{-8}\right.$ to $\left.10^{-5} \mathrm{M}\right)$ caused an $\sim 90-$ 95\% maximal relaxation of aortae taken from both sham and CS-exposed mice with no statistically significant difference observed between groups (Figure 1B), suggesting CS exposure has no effect on vascular smooth muscle function.

CS exposure drives pulmonary immune cell infiltration, pro-inflammatory and oxidative stress gene expression

CS exposure caused a significant increase in the total number of immune cells infiltrating the lungs when compared to sham-exposed control mice (Figure $2 \mathrm{~A}, p<0.05$ ). This increase in total cell number was attributed to a significant increase in macrophages, neutrophils and lymphocytes) (Figures $2 \mathrm{~B}, \mathrm{C} \& \mathrm{D}, p<0.05$ ) .

To better understand the mechanism(s) underlying the increased BALF inflammation, the pulmonary expression of both pro-inflammatory and oxidative stress genes was examined. CS exposure caused a significant increase in mRNA expression of the pro-inflammatory cytokines TNF- $\alpha$ (2.2-fold) and IL-6 (4.7-fold), as well as the key oxidative stress enzyme NOX-2 (1.5-fold; Figures 2, F \& G, $p<0.05$ ).

Endothelial nitric oxide synthase expression is downregulated due to increased vascular ROS in COPD

Given that chronic CS exposure causes endothelium-dependent vascular dysfunction, we then went on to investigate the potential underlying mechanism driving this impaired vascular function. Endothelial expression of the key vascular tone regulator eNOS and a marker of oxidative stress, 3-nitrotyrosine (3-NT), were quantified. CS exposure significantly reduced expression of eNOS by $\sim 70 \%$ (Figure $3 \mathrm{~A}, p<0.05$ ). In addition, the level of 3-NT expression was significantly upregulated by $\sim 3.2$ fold following CS exposure (Figure $3 \mathrm{~B}, p<0.05$ ), indicative of enhanced vascular oxidative stress which may be responsible for the reduced expression of eNOS.

Ebselen prevents CS-induced endothelial dysfunction

As chronic CS exposure caused significant lung inflammation as well as heightened lung and vascular oxidative stress, we sought to investigate the effect of antioxidant treatment on vascular endothelial function. Sham + Veh-treated mice showed an $\sim 90 \% \mathrm{R}_{\max }$, while $\mathrm{CS}+$ Veh-treated mice showed an $\sim 40 \% \mathrm{R}_{\max }$ to $\mathrm{ACh}$ (Figure 4A), confirming CS-induced endothelial dysfunction as also demonstrated in Figure 1. CS-exposed mice treated with ebselen were completely protected from the CS-induced endothelial dysfunction evidenced by the maximal relaxation of $\sim 90 \%$ to ACh. In addition, ebselen did not affect vascular endothelial function in sham-exposed mice (Figure 4A), suggesting its protective effects are specific to CS exposure. Like the data in Figure 1, smooth muscle relaxant responses to SNP were unaltered irrespective of CS exposure or ebselen treatment (Figure 4B).

Ebselen reduces pulmonary immune cell infiltration but has no effect on pro-inflammatory and oxidative stress gene expression

Given that ebselen was able to protect against CS-induced vascular dysfunction, we next sought to determine the effect of ebselen on CS-induced lung inflammation. Consistent with Figure 2, CS caused a significant increase in BALF total cells, macrophages, neutrophils and lymphocytes (Figure 5A-D). Interestingly, ebselen significantly reduced CS-induced increases in BALF total cell and neutrophil counts but not macrophage and lymphocyte numbers (Figure 5A-D). Moreover, while CS exposure increased TNF- $\alpha$ and NOX-2 mRNA expression in the lungs, no detectable attenuations were found with ebselen administration (Figure $5 \mathrm{E} \mathrm{\&} \mathrm{F}$ ). Ebselen administration also appears to be ineffective in preserving lung Gpx-1 mRNA expression against CS exposure (Figure 5G) but rather mimics its antioxidant activity.

Ebselen diminishes CS-induced vascular oxidative stress and downregulation of eNOS 
We next sought to define the underlying mechanism by which ebselen prevented CS-induced endothelial dysfunction. Mice chronically exposed to CS had increased vascular endothelial oxidative stress as measured by 3 -NT expression (Figure $6, p<0.05$ ). However, ebselen administration significantly reduced CS-induced increases in 3-NT expression to baseline sham levels (Figure $6, p<0.05$ ). Moreover, ebselen treatment completely prevented the downregulation of eNOS as a result of CS-exposure (Figure 7, $p<0.05$ ). Collectively, these findings suggest that ebselen reduced CS-induced vascular oxidative stress and subsequently prevented the downregulation of eNOS within the thoracic aorta.

\section{Discussion}

In the present study, the mechanisms underlying systemic endothelial dysfunction, the key driver of comorbid CVD-associated mortality are revealed and the impact of CS-exposure on blood vessel function in our preclinical murine model of COPD were examined. We found that 8-weeks of CS exposure increased immune cell infiltration into the lung resulting in significant pulmonary inflammation and oxidative stress. Pulmonary immune cell infiltration is believed to be the underlying factor driving increased NOX-2 expression and ROS formation. Studies from our group have shown that mice treated with influenza A virus had increased levels of ROS production in the lungs as a consequence of increased pulmonary inflammation (macrophages and neutrophils) and NOX-2 expression (To et al., 2017; Vlahos \& Selemidis, 2014; Vlahos, Stambas, Bozinovski, Broughton, Drummond \& Selemidis, 2011). In this study we also showed increased NOX-2 expression in the lung which is presumably a result of increased pulmonary macrophage and neutrophil numbers in response to cigarette smoke. We also found a significant increase in the expression of the pro-inflammatory mediators TNF- $\alpha$ and IL- 6 in response to CS, which is consistent with our previously published work (Hansen et al., 2013; Vlahos et al., 2006). TNF- $\alpha$ is largely secreted by stimulated macrophages (e.g. in response to CS), driving the inflammatory response and intracellular ROS production, whilst downregulating antioxidant activity (Mukhopadhyay, Hoidal \& Mukherjee, 2006). IL-6 has also been implicated in the pathophysiology of pulmonary diseases, thus, these pro-inflammatory mediators may contribute to pulmonary inflammation and reduced lung function observed in COPD patients (Rincon \& Irvin, 2012),.

Having established that mice exposed chronically to CS had pulmonary inflammation, enhanced oxidative stress and systemic inflammation, we then proceeded to investigate whether this may impact on blood vessel function. It was clear from the present study that CS exposure significantly impaired vasodilation of mouse thoracic aorta to $\mathrm{ACh}$ and that this was specifically attributed to endothelial dysfunction without affecting smooth muscle function. However, it was unclear if this was a secondary result of pulmonary and systemic inflammation or a direct result of CS-induced ROS on the vasculature, and therefore further experimentation is required to ascertain a definitive explanation. Nevertheless, this CS-induced endothelial dysfunction may be a critical link between the heightened risk of CVD and related mortality that claims the lives of $~ 50 \%$ of COPD patients, as well as current and ex-smokers.

It has been well characterised in models of diabetes mellitus (DM) that increased vascular oxidative stress is the key driver of endothelial dysfunction seen in diabetic complications (de Haan \& Cooper, 2011; Kolluru, Bir \& Kevil, 2012; Rask-Madsen \& King, 2007; Shenouda et al., 2011; Tabit, Chung, Hamburg \& Vita, 2010). The excess blood-glucose from DM drives the formation of ROS; such as superoxide, which reduces the bioavailability of NO in the vascular wall (Tabit, Chung, Hamburg \& Vita, 2010). The increased oxidative burden in patients with DM resembles that seen in patients with COPD, as both may lead to posttranslational modification of eNOS and increase vascular oxidative stress, thereby resulting in endothelial dysfunction (de Haan \& Cooper, 2011). Having shown that CS causes endothelium-dependent vascular dysfunction, we next investigated whether this was attributed to changes in eNOS expression. We found that CS caused an $~ 60 \%$ reduction in the expression of eNOS, which would likely lead to a drastic reduction in the production of the key vasodilator NO and reduced NO bioavailability, a hallmark feature of CVD. With studies like that of de Hann et.al showing that eNOS can undergo oxidative modification under highly oxidative environments (de Haan \& Cooper, 2011), vascular expression levels of peroxynitrite were therefore analysed using 3-NT, which specifically detects the conversion of superoxide radical and NO to ONOO-. In agreement with this, the present study also found enhanced vascular oxidative burden following exposure to 
CS, which in turn may promote endothelial dysfunction via post-translational modifications of eNOS and ablating the bioavailability of NO.

Given the significant role of oxidative stress in COPD and this study, we reasoned whether the administration of ebselen, an antioxidant drug which has shown promising results in the context of DM-induced vascular complications through its free radical scavenging activity, could prevent CS-induced vascular dysfunction. Moreover, de Haan et.alhave proposed that deficiencies in the antioxidant GPX and an enhanced oxidative burden promotes endothelial dysfunction leading to DM-related micro- and macrovascular complications (de Haan \& Cooper, 2011). As such, targeted antioxidant replenishment therapy using GPX-mimetics (i.e. ebselen) may be effective in reducing the cardiovascular manifestations in disease states such as DM. de Haan et al . also noted that, an increase in ROS within the vascular endothelium, is one of the most significant factors in NO reduction (de Haan \& Cooper, 2011).

In the present study we showed that ebselen completely prevented endothelial dysfunction induced by CSexposure. We found that ebselen significantly reduced CS-induced endothelial 3-NT expression and that ebselen was able to prevent the loss of aortic eNOS by CS-exposure. It is well established that under normal physiological conditions, stimulation of the vascular endothelium drives the production of NO, diffusing to the surrounding cells, in particular the underlying vascular smooth muscle cells inducing vasodilation, as well as preventing the adhesion and migration of leukocytes and platelets into/onto the arterial wall, thereby maintaining normal vascular function (Versari, Daghini, Virdis, Ghiadoni \& Taddei, 2009). However, vascular oxidative stress may evoke endothelial damage which may significantly impair NO bioavailability and eNOS activity within the vascular endothelium (de Haan \& Cooper, 2011; Versari, Daghini, Virdis, Ghiadoni \& Taddei, 2009). This would post significant risk for the development of CVDs, and mortality in DM patients. This data suggests that modulation of oxidative stress may be beneficial in the clinical treatment of CVD in the context of COPD.

Consistent with our previous studies, ebselen significantly reduced CS-induced BALF inflammation which was largely attributed to a reduction in neutrophilic infiltration (Duong, Seow, Bozinovski, Crack, Anderson \& Vlahos, 2010; Oostwoud et al., 2016). Excess neutrophils play a detrimental role in COPD particularly during periods of acute exacerbation, as they can directly induce protease mediated tissue damage, that has been directly correlated to worsening of emphysema in these patients (Oostwoud et al., 2016; Pesci et al., 1998). MMP activation drives a loss of lung integrity and an increase in permeability which may facilitate the spill over of proinflammatory mediators into the systemic circulation.

It was interesting to note that CS-induced whole lung gene expression of the pro-inflammatory mediator $\mathrm{TNF} \alpha$ and the oxidative stress enzyme NOX-2 were not reduced by ebselen pre-treatment. Although not investigated in the present study, it would be worth exploring whether TNF- $\alpha$ protein expression is altered following ebselen administration. Similarly, it would be worth investigating whether ebselen can directly impede the activity of the regulatory p47phox and other subunits of the NOX-2 enzyme ultimately reducing superoxide production, as this has been previously shown (Smith et al., 2012). The ROS scavenging properties of ebselen within the lung have also been established in the context of asthma, with Zhang et.al showing that following ovalbumin challenge, guinea pigs showed significantly enhanced pulmonary superoxide and hydrogen peroxide concentration, which was ablated in ebselen treated animals (Zhang et al., 2002), reinforcing the powerful antioxidant properties of ebselen. A study by Yatmaz et.al showed that genetic depletion of Gpx-1 causes a significant increase in BALF cellularity in response to influenza infection (HKx31) when compared to wild type control mice (Yatmaz et al., 2013). Interestingly, administration of ebselen $\left(10 \mathrm{mg} / \mathrm{kg}^{-1}\right)$ abolished this viral induced immune cell recruitment to the lung, however, it did not reduce either the protein or mRNA expression of pro-inflammatory cytokines and chemokines (Yatmaz et al., 2013). Nevertheless, the protective effects of ebselen in CS-induced lung inflammation and oxidative stress are promising however, they yet to be determined thoroughly.

It has been shown that there is upregulation of Gpx-1 gene expression in the lungs of smokers (Barnes \& Celli, 2009), which may be a compensatory antioxidant mechanism in response to noxious effects of CS. Conversely, smokers and patients with established COPD have reduced Gpx activity (James \& Wenzel, 2007; 
Versari, Daghini, Virdis, Ghiadoni \& Taddei, 2009; Vlahos et al., 2006), contributing to an overexuberant oxidative burden in the lungs of these patients (Geraghty et al., 2013). In the present study we found that whole lung Gpx-1 mRNA expression was significantly downregulated in mice exposed to CS irrespective of ebselen treatment, further reinforcing that loss of Gpx would increase lung oxidative stress and inflammation. Blunted Gpx expression has also been implicated as a contributing factor in driving endothelial dysfunction, inducing apoptosis and promoting atherosclerosis systemically (Geraghty et al., 2013).

Ebselen treatment has shown promising effects on the vasculature in this study by completely preventing endothelial dysfunction in CS-exposed mice as well as reducing BALF cellularity attributed to neutrophilic infiltration. It has been established that eNOS can undergo oxidative modification as a direct result of the heightened oxidative burden in smokers (Arunachalam, Yao, Sundar, Caito \& Rahman, 2010; Edirisinghe \& Rahman, 2010; Li \& Forstermann, 2014; Zhang, Venardos, Chin-Dusting \& Kaye, 2006). Findings from this study showed that eNOS expression as quantified through immunofluorescent staining was significantly downregulated as a result of CS exposure. However, pre-treatment with ebselen prevented CS-induced downregulation of eNOS and was the likely mechanism by which ebselen restored vascular function in CSexposed mice. It was also interesting to note that ebselen significantly reduced CS-induced 3-NT staining in the thoracic aorta indicating that ebselen completely prevented enhanced oxidative stress within the vascular endothelium, leading to sustained eNOS levels and normal vascular function in CS-exposed ebselen treated mice.

In conclusion, we found that chronic CS exposure in mice causes endothelial dysfunction, as a direct result of enhanced vascular oxidative stress leading to a downregulation of eNOS. In addition, ebselen administration significantly reduced CS-induced lung inflammation and vascular oxidative stress leading to restored vascular endothelial function in CS-exposed mice. Collectively, the data from the present study suggest that ebselen may be a novel therapeutic in the treatment of both the pulmonary manifestations and cardiovascular comorbidities associated with cigarette smoke-induced COPD.

\section{References}

Arunachalam G, Yao H, Sundar IK, Caito S, Rahman I (2010). SIRT1 regulates oxidant- and cigarette smoke-induced eNOS acetylation in endothelial cells: Role of resveratrol. Biochem Biophys Res Commun 393: 66-72.

Austin V, Crack PJ, Bozinovski S, Miller AA, Vlahos R (2016). COPD and stroke: are systemic inflammation and oxidative stress the missing links? Clin Sci (London, England : 1979) 130: 1039-1050.

Azad GK, Tomar RS (2014). Ebselen, a promising antioxidant drug: mechanisms of action and targets of biological pathways. Mol Biol Rep 41: 4865-4879.

Barnes PJ, Celli BR (2009). Systemic manifestations and comorbidities of COPD. Eur Respir J 33: 11651185.

Bartalis J, Chan WG, Wooten JBJAc (2007). A new look at radicals in cigarette smoke. Anal Chem 79: 5103-5106.

Bernardo I, Bozinovski S, Vlahos R (2015). Targeting oxidant-dependent mechanisms for the treatment of COPD and its comorbidities. Pharmacol Ther 155: 60-79.

Brassington K, Selemidis S, Bozinovski S, Vlahos R (2019). New frontiers in the treatment of comorbid cardiovascular disease in chronic obstructive pulmonary disease. Clin Sci (London, England: 1979) 133: 885-904.

Chan CK, Vanhoutte PMJAPSB (2013). Hypoxia, vascular smooth muscles and endothelium. Acta Pharm Sin B 3: 1-7.

Chan SM, Cerni C, Passey S, Seow HJ, Bernardo I, van der Poel C, et al. (2020). Cigarette smoking exacerbates skeletal muscle injury without compromising its regenerative capacity. Am J Respir Cell Mol 
Biol 62(2):217-230.

Chew P, Yuen DYC, Stefanovic N, Pete J, Coughlan MT, Jandeleit-Dahm KA, et al. (2010). Antiatherosclerotic and renoprotective effects of ebselen in the diabetic apolipoprotein E/GPx1-double knockout mouse. Diabetes 59: 3198-3207.

Crack PJ, Taylor JM, Flentjar NJ, De Haan J, Hertzog P, Iannello RC, et al. (2001). Increased infarct size and exacerbated apoptosis in the glutathione peroxidase-1 (Gpx-1) knockout mouse brain in response to ischemia/reperfusion injury. J Neurochem 78:1389-1399.

de Haan JB, Cooper ME (2011). Targeted antioxidant therapies in hyperglycemia-mediated endothelial dysfunction. Front Biosci (Scholar edition) 3: 709-729.

Duong C, Seow HJ, Bozinovski S, Crack PJ, Anderson GP, Vlahos R (2010). Glutathione peroxidase-1 protects against cigarette smoke-induced lung inflammation in mice. Am J Physiol Lung Cell Mol Physiol 299:L425-433.

Edirisinghe I, Rahman I (2010). Cigarette smoke-mediated oxidative stress, shear stress, and endothelial dysfunction: role of VEGFR2. Ann N Y Acad Sci 1203: 66-72.

Endemann DH, Schiffrin EL (2004). Endothelial dysfunction. J Am Soc Nephrol 15: 1983-1992.

Geraghty P, Hardigan AA, Wallace AM, Mirochnitchenko O, Thankachen J, Arellanos L, et al. (2013). The glutathione peroxidase 1-protein tyrosine phosphatase 1B-protein phosphatase $2 \mathrm{~A}$ axis. A key determinant of airway inflammation and alveolar destruction. Am J Respir Cell Mol Biol 49: 721-730.

Hansen MJ, Chen H, Jones JE, Langenbach SY, Vlahos R, Gualano RC, et al. (2013). The lung inflammation and skeletal muscle wasting induced by subchronic cigarette smoke exposure are not altered by a high-fat diet in mice. PLoS One 8: e80471.

James AL, Wenzel S (2007). Clinical relevance of airway remodelling in airway diseases. Eur Respir J 30: $134-155$.

Kluchova Z, Petrasova D, Joppa P, Dorkova Z, Tkacova R (2007). The association between oxidative stress and obstructive lung impairment in patients with COPD. Physiol Res 56: 51-56.

Kolluru GK, Bir SC, Kevil CG (2012). Endothelial dysfunction and diabetes: effects on angiogenesis, vascular remodeling, and wound healing. Int J Vasc Med 2012: 918267.

Li H, Forstermann U (2014). Pharmacological prevention of eNOS uncoupling. Curr Pharm Des 20: 35953606 .

Mukhopadhyay S, Hoidal JR, Mukherjee TK (2006). Role of TNFalpha in pulmonary pathophysiology. Resp Res 7: 125 .

Oostwoud LC, Gunasinghe P, Seow HJ, Ye JM, Selemidis S, Bozinovski S, et al. (2016). Apocynin and ebselen reduce influenza A virus-induced lung inflammation in cigarette smoke-exposed mice. Sci Rep 6: 20983.

Pesci A, Balbi B, Majori M, Cacciani G, Bertacco S, Alciato P, et al. (1998). Inflammatory cells and mediators in bronchial lavage of patients with chronic obstructive pulmonary disease. Eur Respir J 12: 380-386.

Rask-Madsen C, King GL (2007). Mechanisms of Disease: endothelial dysfunction in insulin resistance and diabetes. Nat Clin Pract Endocrinol Metab 3: 46-56.

Rincon M, Irvin CG (2012). Role of IL-6 in asthma and other inflammatory pulmonary diseases. Int J Biol Sci 8: 1281-1290. 
Santos M, Oliveira A, Viegas-Crespo A, Vicente L, Barreiros A, Monteiro P, et al. (2004). Systemic markers of the redox balance in chronic obstructive pulmonary disease. Biomarkers 9: 461-469.

Sarker KP, Biswas KK, Rosales JL, Yamaji K, Hashiguchi T, Lee KY, et al. (2003). Ebselen inhibits NOinduced apoptosis of differentiated PC12 cells via inhibition of ASK1-p38 MAPK-p53 and JNK signaling and activation of p44/42 MAPK and Bcl-2. J Neurochem 87: 1345-1353.

Sarma BK, Mugesh G (2008). Antioxidant activity of the anti-inflammatory compound ebselen: a reversible cyclization pathway via selenenic and seleninic acid intermediates. Chemistry 14: 10603-10614.

Shenouda SM, Widlansky ME, Chen K, Xu G, Holbrook M, Tabit CE, et al. (2011). Altered mitochondrial dynamics contributes to endothelial dysfunction in diabetes mellitus. Circulation 124: 444-453.

Sin DD, Anthonisen NR, Soriano JB, \& Agusti AG (2006). Mortality in COPD: Role of comorbidities.Eur Respir J 28: 1245-1257.

Sin DD, Man SF (2005). Chronic obstructive pulmonary disease as a risk factor for cardiovascular morbidity and mortality. Proc Am Thorac Soc 2: 8-11.

Smith SM, Min J, Ganesh T, Diebold B, Kawahara T, Zhu Y, et al.(2012). Ebselen and congeners inhibit NADPH oxidase 2-dependent superoxide generation by interrupting the binding of regulatory subunits. Chem Biol 19: 752-763.

Tabit CE, Chung WB, Hamburg NM, Vita JA (2010). Endothelial dysfunction in diabetes mellitus: molecular mechanisms and clinical implications. Rev Endocr Metab Disord 11: 61-74.

Takasago T, Peters EE, Graham DI, Masayasu H, Macrae IM (1997). Neuroprotective efficacy of ebselen, an anti-oxidant with anti-inflammatory actions, in a rodent model of permanent middle cerebral artery occlusion. Br J Pharmacol 122: 1251-1256.

Tkacova R, Kluchova Z, Joppa P, Petrasova D, \& Molcanyiova A (2007). Systemic inflammation and systemic oxidative stress in patients with acute exacerbations of COPD. Respir Med 101: 1670-1676.

The Lung Foundation. COPD: The Statistics. Available from http://lungfoundation.com.au/healthprofessionals/clinical-resources/copd/copd-the-statistics/. [Accessed: 15.3.2017].

To EE, Vlahos R, Luong R, Halls ML, Reading PC, King PT, et al.(2017). Endosomal NOX2 oxidase exacerbates virus pathogenicity and is a target for antiviral therapy. Nat Commun 8: 69 .

Versari D, Daghini E, Virdis A, Ghiadoni L, Taddei S (2009). Endothelial dysfunction as a target for prevention of cardiovascular disease. Diabetes Care 32 Suppl 2: S314-321.

Vlahos R, Bozinovski S (2014). Recent advances in pre-clinical mouse models of COPD. Clin Sci (London, England : 1979) 126: 253-265.

Vlahos R, Bozinovski S, Chan SPJ, Ivanov S, Lindén A, Hamilton JA, et al. (2010). Neutralizing granulocyte/macrophage colony-stimulating factor inhibits cigarette smoke-induced lung inflammation. Am J Respir Crit Care Med 182: 34-40.

Vlahos R, Bozinovski S, Jones JE, Powell J, Gras J, Lilja A, et al. (2006). Differential protease, innate immunity, and NF-kappaB induction profiles during lung inflammation induced by subchronic cigarette smoke exposure in mice. Am J Physiol Lung Cell Mol Physiol 290: L931-945.

Vlahos R, Selemidis S (2014). NADPH oxidases as novel pharmacologic targets against influenza A virus infection. Mol pharmacol 86:747-759.

Vlahos R, Stambas J, Bozinovski S, Broughton BRS, Drummond GR, Selemidis S (2011). Inhibition of Nox2 Oxidase Activity Ameliorates Influenza A Virus-Induced Lung Inflammation. PLoS Pathog 7: e1001271. 
The World Health Organisation. The top 10 causes of death.Available from http://www.who.int/newsroom/fact-sheets/detail/the-top-10-causes-of-death..

Yatmaz S, Seow H, Gualano R, Wong Z, Stambas J, Selemidis S, et al. (2013). Glutathione Peroxidase-1 Reduces Influenza A Virus-Induced Lung Inflammation. Am J Respir Cell Mol Biol 48: 17-26.

Zhang M, Nomura A, Uchida Y, Iijima H, Sakamoto T, Iishii Y, et al. (2002). Ebselen suppresses late airway responses and airway inflammation in guinea pigs. Free radic biol Med 32: 454-464.

Zhang WZ, Venardos K, Chin-Dusting J, Kaye DM (2006). Adverse effects of cigarette smoke on NO bioavailability: role of arginine metabolism and oxidative stress. Hypertension 48: 278-285.

Figure Legends

Figure 1: CS exposure causes endothelial dysfunction in the thoracic aorta. Cumulative concentration response curves to $(\mathbf{A})$ acetylcholine and $(\mathbf{B})$ sodium nitroprusside $\left(1 \times 10^{-8} \mathrm{M}\right.$ to $\left.1 \times 10^{-5} \mathrm{M}\right)$ to assess both endothelial-dependent and smooth muscle-dependent vasodilatory responses in mouse thoracic aorta ( $\mathrm{n}=8$ /group) following either chronic CS or sham exposure, respectively. Results are expressed as mean percentage relaxation relative to precontraction \pm SEM. * indicates statistical significance $(p<0.05)$ between data sets by two-way ANOVA with Tukey's multiple comparisons.

Figure 2: Chronic CS exposure increases BAL fluid cellularity and enhances both lung proinflammatory and oxidative stress mediator gene expression. The lungs of mice exposed to CS were lavaged for the assessment of total cells (A), macrophages (B), neutrophils (C) and lymphocytes (D) (n=10). Whole lungs excised from mice were then used to measure mRNA expression by RT-qPCR of TNF $\alpha$ (E) $(\mathrm{n}=10), \mathrm{IL}-6(\mathrm{~F})(\mathrm{n}=10)$ and NOX-2 $(\mathrm{G})(\mathrm{n}=9)$. Gene expression data are expressed as fold change relative to the sham group. All data are expressed as mean + SEM and were analysed by students unpaired t-test with significance being denoted by $*(p<0.05)$ between treatment groups.

Figure 3 Immunofluorescent staining of endothelial nitric oxide synthase and 3-nitrotyrosine in the thoracic aorta of mice exposed to 8 weeks of CS or room air. Immunofluorescent quantification of eNOS and 3-NT expression in either sham or 8-week CS-exposed mice. Green staining indicates the presence of eNOS (A) or 3-NT (B) and blue staining denotes the nuclear counterstain, DAPI (4', 6-diamidino2- phenylindole, dilactate). Representative photographs of immunofluorescent staining in (A) sham-exposed or (B) CS-exposed mice. Expression of eNOS ( $\mathrm{n}=5-6$ mice per group) and 3-NT ( $\mathrm{n}=5$ mice per group) both normalised to the relative negative control and expressed as fold percentage change relative to the sham-treated group. Scale bar represents $50 \mu \mathrm{M}$. Data expressed as mean + SEM and analysed by students unpaired t-test with significance being denoted by; * indicating $p<0.05$.

Figure 4 Ebselen prevents CS-induced endothelial dysfunction. Cumulative concentration response curves to acetylcholine (A) and sodium nitroprusside (B) $\left(1 \times 10^{-8} \mathrm{M}\right.$ to $\left.1 \times 10^{-5} \mathrm{M}\right)$ to assess endothelial and smooth muscle-dependent vasodilation in mouse thoracic aorta $(n=6)$ following either chronic CS or sham exposure in either ebselen or vehicle-treated mice, respectively. Results are expressed as mean percentage relaxation relative to pre-constriction \pm SEM. $*$ indicates statistical significance $(p<0.05)$ between data sets by two-way ANOVA with Tukey's multiple comparisons.

Figure 5: Effect of chronic CS and ebselen treatment on BAL fluid cellularity and pulmonary pro-inflammatory and oxidative stress gene expression. The lungs of mice exposed to CS were lavaged for the assessment of total cells (A), macrophages (B), neutrophils (C) and lymphocytes (D) $(n=10)$. Whole lungs excised from mice were then used to measure mRNA expression by RT-qPCR of TNF $\alpha$ (E), NOX-2 $(\mathrm{F})$ and GPX-1(G) $(\mathrm{n}=10)$. Responses are expressed as fold change relative to the sham + vehicle-treated group, post normalisation to GAPDH (housekeeping gene). All data are expressed as mean + SEM and were analysed by 2 -way ANOVA and Tukey's post hoc analysis with significance being denoted by * $(p<0.05)$ between treatment groups.

Figure 6: Immunofluorescent staining of 3-Nitrotyrosine in the thoracic aorta of mice exposed 
to chronic CS and treated with ebselen.Immunofluorescent quantification of 3-NT expression in either sham or 8-week CS-exposed mice that were treated with either vehicle or ebselen. Green staining indicates the presence of 3-NT specific and blue staining denotes the nuclear counterstain, DAPI (4', 6-diamidino-2phenylindole, dilactate). Representative photographs of immunofluorescent staining in (A) sham-exposed vehicle treated $(n=5),(B)$ sham-exposed ebselen treated $(n=5),(C)$ CS-exposed vehicle treated $(n=4)$ and (D) CS-exposed ebselen treated mice $(\mathrm{n}=6)$. Expression of 3 -NT normalised to the negative control and expressed as fold percentage change relative to the sham vehicle-treated group. Scale bar represents 50 $\mu \mathrm{M}$. Data expressed as mean + SEM and analysed by two-way ANOVA with Tukey's multiple comparisons; significance is denoted by; * indicating $p<0.05$.

Figure 7: Immunofluorescent staining of endothelial nitric oxide synthase in the thoracic aorta of mice exposed chronically to CS and treated with ebselen Immunofluorescent staining of eNOS in either sham or 8-week CS-exposed mice with or without ebselen administration. Green staining detects the presence of eNOS and Blue staining denotes the nuclear counterstain, DAPI (4', 6-diamidino-2- phenylindole, dilactate). Representative photographs of immunofluorescent staining in (A) sham-exposed vehicle treated $(n=4),(B)$ sham-exposed ebselen treated $(n=4),(C)$ CS-exposed vehicle treated $(n=7)$ and (D) CS-exposed ebselen treated mice $(n=7)$. Expression of eNOS normalised to the negative control and expressed as fold percentage change relative to the sham vehicle-treated group. Scale bar represents 50 $\mu$ M. Data expressed as mean \pm SEM and analysed by two-way ANOVA with Tukey's multiple comparisons; significance is denoted by $*(p<0.05)$.

\section{Hosted file}

Brassington K BJP Final Figures Submitted 3 September 2020.pptx available at https: //authorea.com/users/356125/articles/479107-ebselen-reduces-cigarette-smoke-inducedvascular-endothelial-dysfunction-in-mice 